\title{
Predição volumétrica por meio da krigagem pontual reduz o esforço de amostragem em inventários florestais pré-corte
}

\author{
Daniel Dantas ${ }^{1 *}$, Luiz Otávio Rodrigues Pinto², Anny Francielly Ataíde Gonçalves ${ }^{3}$, Marcela de Castro Nunes \\ Santos Terra ${ }^{4}$, Natalino Calegario ${ }^{5}$
}

DOI: https://doi.org/10.35699/2447-6218.2020.15927

\begin{abstract}
Resumo
O objetivo deste trabalho foi avaliar o desempenho de um estimador geoestatístico para estimar a volumetria de um povoamento de eucalipto com 8 anos de idade considerando diferentes intensidades amostrais. A base de dados foi proveniente de inventários florestais pré-corte. A intensidade amostral foi de uma parcela a cada 5 ha aproximadamente, totalizando 220 parcelas inventariadas em campo. Os dados foram divididos em dois grupos: $80 \%$ para ajuste dos semivariogramas e $20 \%$ para validação. Dentre os dados destinados aos ajustes, foram selecionadas parcelas que representassem quatro diferentes intensidades amostrais $(1: 5,1: 10,1: 15$ e 1:20), e posteriormente, ajustados os modelos de semivariogramas. Os modelos foram então utilizados para estimar o volume das parcelas destinadas à validação. Os resultados permitiram inferir que o modelo esférico ajustado para as diferentes intensidades amostrais apresentou desempenho satisfatório e próximos entre si, com erros inferiores a $10 \%$. O menor valor foi apresentado na intensidade amostral de 1:5, 7,33 \%, e o maior na intensidade de 1:20, 8,90\%, uma diferença de apenas 1,57 $\%$. Assim sendo, foi possível concluir que o estimador geoestatístico permitiu a redução da intensidade amostral em inventários que antecedem o corte de povoamentos clonais de Eucalyptus, sem grandes perdas na precisão.
\end{abstract}

Palavras-chave: Semivariograma. Inventário Florestal. Intensidade amostral.

\section{Volumetric prediction through punctual kriging reduces sampling effort in pre-cut forest inventories}

\begin{abstract}
The objective of this work was to evaluate the performance of a geostatistical estimator to estimate the wood volume per hectare of an eucalyptus stand, considering different sample intensities. The database was derived from pre-cut forest inventories, with a sampling intensity of one plot per 4.95 ha (approximately, 1:5), totaling 220 plots. Data were splitted into two groups: $80 \%$ for semivariograms fits and $20 \%$ for validation. Sampling units representing four different sample intensities (1:5, 1:10, 1:15 and 1:20) were selected and the semivariograms models were fitted. The models were then used to estimate the volumes per hectare of the parcels intended for validation. It was verified that the spherical models adjusted for the different sample intensities presented satisfactory and similar performances with each other, with mean relative errors lower than $10 \%$. The lowest value was presented in the sampling intensity of $1: 5,7.33 \%$, and the highest in the intensity of $1: 20,8.90 \%$. An average relative error difference of only $1.57 \%$.
\end{abstract}

\footnotetext{
${ }^{1}$ Universidade Federal de Lavras. Departamento de Ciências Florestais. Lavras, MG, Brasil. http://orcid.org/0000-0002-7928-9155

${ }^{2}$ Universidade Federal de Lavras. Departamento de Ciências Florestais. Lavras, MG, Brasil. http://orcid.org/0000-0003-2854-2883

${ }^{3}$ Universidade Federal de Lavras. Departamento de Ciências Florestais. Lavras, MG, Brasil. http://orcid.org/0000-0001-5189-0259

${ }^{4}$ Universidade Federal de Lavras. Departamento de Ciências Florestais. Lavras, MG, Brasil. http://orcid.org/0000-0003-4646-2414

${ }^{5}$ Universidade Federal de Lavras. Departamento de Ciências Florestais. Lavras, MG, Brasil. http://orcid.org/0000-0001-8323-1223

*Autor para correspondência: dantasdaniel12@yahoo.com.br
}

Recebido para publicação em 30 de outubro de 2019. Aceito para publicação em 13 de maio de 2020.

e-ISSN: 2447-6218 / ISSN: 2447-6218 / (c) 2009, Universidade Federal de Minas Gerais, Todos os direitos reservados. 
Dantas, D. et al.

Therefore, it is possible to reduce the sample intensity in pre-cut inventories for Eucalyptus stands, without great losses in accuracy, with the use of point kriging to obtain the volume of wood per hectare in non-sampled points.

Keywords: Semivariogram. Forest inventory. Geostatistical model.

\section{Introdução}

Uma das informações de maior importância para o conhecimento do potencial de uma floresta, em uma dada região, é a variável volume de madeira, sendo imprescindível para o planejamento, exploração ou manejo sustentável de florestas. O volume individual é um ponto de partida para a avaliação do conteúdo do estoque de madeira em um povoamento florestal (Machado et al., 2002) e serve como base para as decisões relacionadas a tratos silviculturais, colheita e transporte da madeira. Assim, é fundamental que a obtenção do volume de madeira seja realizada de maneira representativa, com baixo custo e exatidão.

A necessidade do desenvolvimento e da utilização de ferramentas estatísticas, capazes de caracterizar a estrutura de variabilidade espacial de um determinado fenômeno, motivou o surgimento da teoria das variáveis regionalizadas ou, simplesmente, geoestatística (Nogueira, 2013), motivado pela constante busca por informações mais detalhadas (Santos et al., 2017). De acordo com Couto et al. (1997), a geoestatística tem grande utilidade para caracterizar e mapear a variação espacial de diversos fenômenos.

Nogueira (2013) afirma que as variáveis regionalizadas apresentam dupla característica: aleatoriedade, uma vez que seus valores numéricos observados podem variar, consideravelmente, de um ponto a outro no espaço e, espacialidade, pois estes valores não são inteiramente independentes, denotando uma aparente continuidade espacial, na qual podem ser captados e modelados matematicamente por técnicas geoestatísticas.

Segundo Mello (2004), a teoria das variáveis regionalizadas pressupõe que cada dado é modelado com uma variável aleatória que pode ser expressa pela soma de três componentes: um componente estrutural, associado a um valor médio constante ou a uma tendência constante; uma componente aleatória, espacialmente correlacionada; e um erro aleatório constante.

Estudos têm indicado a aplicabilidade e eficiência da geoestatística na modelagem espacial de variáveis florestais, como diâmetro e índice de sítio (Sena et al., 2019) e propriedades químicas do solo (Lima et al., 2017). Raimundo et al. (2017) estudaram a utilização da geoestatística para garantir a representatividade da amostra em um inventário florestal contínuo (IFC) e observaram que a modelagem de índice de sites com o banco de dados reduzido teve a mesma precisão quando o banco de dados completo foi usado. A geoestatística melhorou a precisão e a confiabilidade das estatísticas do
IFC e permitiu a diminuição da intensidade da amostragem. Lundgren et al. (2016) verificaram se a estimativa do volume individual de madeira por árvore realizada pela krigagem é influenciada pelo tipo de amostragem. Onze tipos de erros foram comparados e ranqueados e a amostragem aleatória apresentou os melhores resultados e a amostragem grade aglomerada, os piores. Ainda que muitos estudos tenham se empenhado em descrever a variabilidade espacial de variáveis florestais, poucos avaliaram a possibilidade de redução da intensidade amostral.

Buscando a otimização dos recursos, a adoção destas técnicas como ferramenta de modelagem no manejo florestal, é a possibilidade de redução do número de medições necessárias, sem perda na qualidade das estimativas. Isso acarretaria uma otimização do tempo e custo dos inventários florestais. Para isso, são necessários estudos que forneçam subsídios para o manejador no processamento de dados de inventário florestal. Neste sentido, o objetivo deste trabalho foi avaliar o desempenho do estimador geoestatístico para estimação da volumetria de um povoamento de eucalipto sob diferentes intensidades amostrais.

\section{Materiais e métodos}

\section{Área de estudo}

A área de estudo contempla 21 unidades de manejo de clones de Eucalyptus spp., localizada nos municípios de Bom Jardim de Minas, Lima Duarte e Arantina, Estado de Minas Gerais, Brasil, totalizando 1.090 ha. O clima da região é do tipo tropical de altitude, do tipo Cwb, segundo a classificação de Köppen, com temperatura média anual de $20,1^{\circ} \mathrm{C}$, com invernos secos e frios, com ocorrência de geadas em algumas áreas, e verões chuvosos com temperaturas moderadamente altas. A precipitação total média anual é de 1456 mm (Alvares et al., 2013).

\section{Coleta de dados}

Os dados utilizados neste estudo foram provenientes de inventários florestais temporários, em que foram mensurados 27.299 indivíduos, distribuídos em 565 parcelas. As parcelas retangulares com área variável de aproximadamente $300 \mathrm{~m}^{2}$ foram distribuídas aleatoriamente, de modo representativo ao longo área, tendo como variáveis numéricas: idade (meses), altura (metros), diâmetro à altura do peito (centímetros), área basal da parcela $\left(\mathrm{m}^{2} \cdot \mathrm{ha}^{-1}\right)$ e índice de sítio $(\mathrm{m})$ (Tabela 1$)$. 
Predição volumétrica por meio da krigagem pontual reduz o esforço de amostragem em inventários florestais pré-corte

Tabela 1 - Estatísticas descritivas dos dados de inventário florestal de Eucalyptus spp.

\begin{tabular}{cccccc}
\hline Variável & Mínimo & Média & Máximo & Desvio Padrão & CV\% \\
\hline H & 5,00 & 21,26 & 31,50 & 3,94 & 18,54 \\
DAP & 5,00 & 14,51 & 26,20 & 2,97 & 20,44 \\
I & 36 & 59 & 78 & 9 & 15,93 \\
G & 6,05 & 17,28 & 34,21 & 5,11 & 29,59 \\
S & 12,50 & 25,84 & 32,50 & 3,27 & 12,67 \\
\hline
\end{tabular}

em que: $\mathrm{H}$ é a altura em metros; DAP é o diâmetro, em centímetros, a 1,30 metros de altura do solo; I é a idade, em meses; G é a área basal, em $\mathrm{m}^{2}$.ha- ${ }^{-1}$; e $\mathrm{S}$ é o índice de sítio, em metros.

Para as árvores que não tiveram alturas mensurada em campo, as mesmas foram estimadas por meio de um modelo hipsométrico não linear de efeito misto (Eq. 1), com a relação funcional:

$$
\left.H_{i j}=f\left(D A P_{i j}, I_{i}, G_{i}, S_{i j}\right) \text { (Eq. } 1\right)
$$

em que: $H_{i j}$ é a altura da i-ésima árvore (m) da j-ésima unidade amostral; $D A P_{i j}$ é o diâmetro à altura do peito da i-th arvore $(\mathrm{cm})$ da j-ésima unidade amostral; $I_{i}$ é a idade da i-th árvore (meses); $G_{i}$ é a área basal da i-th parcela $\left(\mathrm{m}^{2} / \mathrm{ha}\right) ; S_{i j}$ é o índice de sítio da j-th unidade amostral contendo a i-th árvore (m); $\varepsilon$ é o erro aleatório (m).

A Tabela 2 indica os parâmetros ajustados e a significância dos parâmetros do modelo não linear (Dantas, 2019), em que todos foram significativos a um nível de 0,05 para o teste $t$-Student. O modelo apresenta um coeficiente de correlação entre valores estimados e observados de 0,9993 e raiz do erro quadrático médio de $0,54 \%$.

Tabela 2 - Significância dos parâmetros fixos ajustados para o modelo hipsométrico não linear misto.

\begin{tabular}{cccccc}
\hline F.V. & Parâmetros & Erro padrão & $\mathbf{t}$ & Pr $(>\mathbf{t})$ & \\
\hline$\beta 0$ & 37,9895 & 0,2069 & 31,4761 & $<0,0000$ & $*$ \\
$\beta 1$ & 0,5669 & 0,0276 & 20,5269 & $<0,0000$ & $*$ \\
$\beta 2$ & $-1430,1869$ & 33,7983 & $-25,1801$ & $<0,0000$ & $*$ \\
$\beta 3$ & $-9,3829$ & 0,5402 & $-17,3691$ & $<0,0000$ & $*$ \\
$\beta 4$ & $-10,8414$ & 0,6561 & $-4,0816$ & $<0,0000$ & $*$ \\
$\beta 5$ & 364,2581 & 19,0100 & 10,7081 & $<0,0000$ & $*$ \\
\hline
\end{tabular}

em que: F.V.: fonte de variação; $\beta_{i}$ : coeficientes da regressão; *: significativo a 0,05 .

Os volumes de madeira das árvores foram estimados por meio do modelo não linear de Schumacher e Hall (1933) (Eq. 2), ajustado com regressão mista, com inclusão de efeitos aleatórios, que foram as variabilidades existentes em cada indivíduo e entre as diferentes unidades de manejo, pelo método dos quadrados mínimos ordinários (Dantas et al., 2020). Para o ajuste foram utilizados dados de cubagem rigorosa, realizada no período de 2010 e 2016, composto por 818 árvores, em que foram mensuradas a altura total $(H t)$, o diâmetro à altura do peito $(D A P)$ e os diâmetros na base das árvores (a $0,1 \mathrm{~m}$ de altura) e nas alturas de $0,5 \mathrm{~m} ; 1 \mathrm{~m} ; 1,5 \mathrm{~m}$ e $2 \mathrm{~m}$; e, a partir desta marca, a cada $2 \mathrm{~m}$. Os volumes individuais foram obtidos pela fórmula de Smalian.

$$
\mathrm{V}_{i}=\beta_{0} * D A P_{i}^{\beta_{1}} * H t_{i}^{\beta_{2}}+\varepsilon_{i} \text { (Eq. 2) }
$$

em que: $V_{i}$ é o volume em $\mathrm{m}^{3}$ da i-ésima árvore; $D A P_{i}$ é o diâmetro, em $\mathrm{cm}$, a 1,30 $\mathrm{m}$ de altura da i-ésima árvore; $H_{t}$ é a altura total, em $\mathrm{m}$, da i-ésima árvore; $\beta_{0}, \beta_{1} e \beta_{2}$ são os parâmetros do modelo; $\varepsilon$ é o erro aleatório $\left(\mathrm{m}^{3}\right)$.
Os parâmetros fixos obtidos com o ajuste do modelo não linear de Schumacher e Hall são apresentados na Tabela 3. O modelo apresenta um coeficiente de correlação entre valores estimados e observados de 0,9831 e raiz do erro quadrático médio de 4,42\% (Dantas et al., 2020).

A equação ajustada foi aplicada aos dados de inventário florestal, em que foram estimados os volumes individuais, posteriormente, estimado o volume de madeira por hectare de cada parcela. Foram selecionadas, dentre os dados utilizados neste estudo, parcelas georreferenciadas por meio de GPS geodésico, oriundas de inventários florestais pré-corte realizados aos 8 anos de idade dos povoamentos, selecionando 220 parcelas, que representam uma intensidade amostral de uma parcela a cada 4,95 ha (aproximadamente, 1:5). 
Dantas, D. et al.

Tabela 3 - Parâmetros fixos do modelo não linear de Schumacher e Hall.

\begin{tabular}{cccccc}
\cline { 2 - 6 } & Parâmetros & Erro padrão & $\mathbf{t}$ & \multicolumn{2}{c}{$\operatorname{Pr}(>\mathbf{t})$} \\
\hline$\beta_{0}$ & $3,4645 \mathrm{e}^{-5}$ & $1,59 \mathrm{e}^{-6}$ & 21,81 & $<0,0000$ & $*$ \\
$\beta_{1}$ & 1,8184 & 0,0200 & 90,85 & $<0,0000$ & $*$ \\
$\beta_{2}$ & 1,1655 & 0,0282 & 41,38 & $<0,0000$ & $*$ \\
\hline
\end{tabular}

em que $\beta_{i}$ : coeficientes da regressão; *: significativo a 0,001 .

As estatísticas descritivas dos dados são apresentadas na Tabela 4.

Tabela 4 - Estatísticas descritivas dos dados de cubagem rigorosa de Eucalyptus spp.

\begin{tabular}{cccccc}
\hline Variável & Mínimo & Médio & Máximo & Desvio padrão & CV\% \\
\hline Volume $\left(\mathrm{m}^{3}\right)$ & 0,0066 & 0,1785 & 0,7752 & 0,1335 & 74,79 \\
DAP $(\mathrm{cm})$ & 4,19 & 13,60 & 27,00 & 4,45 & 32,72 \\
HT $(\mathrm{m})$ & 8,10 & 21,82 & 33,08 & 5,10 & 23,37 \\
\hline
\end{tabular}

em que: DAP é o diâmetro a 1,30 de altura do solo; HT é a altura total.

\section{Análise exploratória}

Inicialmente, foi realizada uma análise exploratória, com a finalidade de observar a tendência espacial dos dados e detectar a presença de outliers. Foram calculadas as estatísticas descritivas de tendência central e de dispersão (média, mediana, moda, desvio padrão e coeficiente de variação), e analisados os comportamentos da variável regionalizada em função da longitude e da latitude. Para verificar a hipótese de normalidade dos dados utilizou-se o teste de Shapiro-Wilk, com um nível de significância de $5 \%$, que testa a hipótese de que a amostra é proveniente de uma população com distribuição normal, ou seja, a característica apresenta distribuição unimodal, em geral simétrica, que se aproxima da distribuição normal (Shapiro e Wilk, 1965).

\section{Intensidade amostral}

Para caracterizar o padrão espacial da variável volume e avaliar a influência da intensidade amostral na precisão das estimativas volumétricas a nível de povoamento, procedeu-se às análises geoestatísticas: análise variográfica e krigagem.

Os dados foram divididos em dois grupos: $80 \%$ para ajuste dos semivariogramas e $20 \%$ para validação. Dentre os dados destinados aos ajustes dos semivariogramas, foram selecionadas unidades amostrais, distribuídas de forma a representar a área e que simulassem quatro diferentes intensidades amostrais: 1 parcela a cada 5 ha (1:5); 1 parcela a cada 10 ha (1:10); 1 parcela a cada 15 ha (1:15) e 1 parcela a cada 20 ha (1:20) (Mello et al., 2009). Para cada intensidade amostral foram calculados o erro padrão da média, erro do inventário e, consequentemente, o intervalo de confiança, de acordo com os estimadores clássicos da Amostragem Casual Simples (ACS) (Cochran, 1977).

\section{Análise variográfica}

Para cada intensidade amostral, foram ajustados três modelos de semivariograma amplamente utilizados na literatura: esférico, exponencial e gaussiano, pelo método da máxima verossimilhança, descrito por Wonnacott e Wonnacott (1972). O processamento foi feito no software R, versão 3.6.1 (R Core Team, 2019), por meio do pacote geoR (Ribeiro Júnior e Diglle, 2001).

Para a escolha do modelo mais adequado a cada conjunto de dados, o critério utilizado foi o menor valor do Critério de Informação Akaike (AIC) (MC Bratney e Webster, 1986; Sakamoto et al., 1986). Segundo Biase e Santana (2012) a utilização deste critério na seleção do modelo é mais indicada, devido à sua maior sensibilidade. Se a diferença entre os modelos for igual ou superior a dois, indica que estes são consideravelmente diferentes entre si (Brunham e Anderson, 2002).

\section{Krigagem}

Após o ajuste e seleção dos modelos de semivariogramas, para cada intensidade amostral, foram realizadas interpolações dos dados pelo método da krigagem ordinária pontual, que considera a dependência espacial e estima valores sem tendência e com variância mínima (Vieira, 2000).

Os modelos de semivariogramas foram então utilizados para predizer a volumetria das parcelas que constituem o conjunto de dados destinados à validação. Esse processamento foi realizado no software ArcGIS 10.1 (ESRI, 2011), por meio da ferramenta kriging (Geoestatitical Analyst). Para avaliação da precisão dos modelos foram calculados os Erros Relativos Médios (ERM\%) entre os volumes de madeira obtidos pelas diferentes intensidades amostrais e o volume de madeira observado, gráficos de distribuição dos volumes de madeira estimados e observados, gráficos de erros percentuais em função 
Predição volumétrica por meio da krigagem pontual reduz o esforço de amostragem em inventários florestais pré-corte

dos valores observados e os coeficientes de correlação entre os volumes de madeira estimados e observados.

\section{Resultados e discussão}

A Tabela 5 apresenta os resultados da análise exploratória dos dados de volumetria do povoamento

Tabela 5 - Estatísticas descritivas dos dados volumétricos do povoamento de eucalipto.

\begin{tabular}{cc}
\hline \multicolumn{2}{c}{ Estatística } \\
\hline Média $\left(\mathrm{m}^{3} \cdot \mathrm{ha}^{-1}\right)$ & 315,45 \\
Mediana $\left(\mathrm{m}^{3} \cdot \mathrm{ha}^{-1}\right)$ & 316,25 \\
Desvio padrão $\left(\mathrm{m}^{3} \cdot \mathrm{ha}^{-1}\right)$ & 77,83 \\
Coeficiente de variação (\%) & 24,36 \\
Teste de Shapiro-Wilk (p-valor) & 0,4268 \\
\hline
\end{tabular}

Segundo Cressie (1993) e Diggle e Ribeiro Júnior (2007), a aplicação da geoestatística não depende de uma distribuição normal dos dados, no entanto, a presença da normalidade permite que sejam feitas inferências com propriedades diferentes, como por exemplo, o uso da Máxima Verossimilhança, como foi feito neste trabalho.

Foram observados dados que podem ser considerados outliers, ou seja, que apresentam comportamento diferente em relação aos demais. Segundo Diggle e Ribeiro Júnior (2000), estes dados provocam forte impacto no ajuste do semivariograma e, portanto, foram removidos da base de dados para que não comprometessem o estudo variográfico. considerando os dados de ajuste e validação. Verificou-se pelo teste de Shapiro-Wilk que os dados se aproximam da distribuição normal $(\mathrm{w}<0,95)$.

Figura 1 - Gráficos exploratórios da variável regionalizada volume por hectare; A: volume em função da latitude; B: volume em função da longitude; C: histograma de frequência.

A)

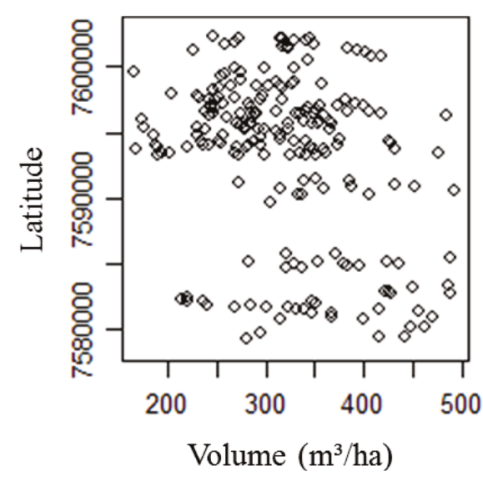

B)

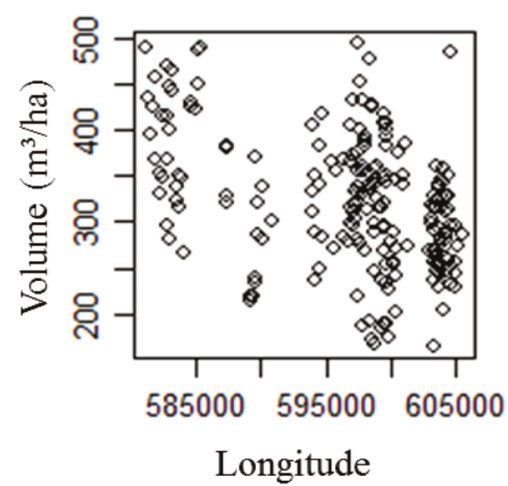

Os principais gráficos a serem observados na análise exploratória de dados são apresentados na Figura 1. Os gráficos A e B mostram o comportamento da variável regionalizada (volume de madeira por hectare) em função da latitude e da longitude. Verificou-se inexistência de tendência para a variável volume nos dois sentidos. Esta é uma característica importante em estudos geoestatísticos, pois indica a possibilidade de se aplicar a Hipótese Intrínseca descrita por Isaaks e Srivastava (1989), Journel e Huijbrets (1978), Ribeiro Júnior (1995) e Vieira (2000). O gráfico C mostra que os dados utilizados têm uma distribuição aproximadamente normal, corroborando o resultado do teste de Shapiro-Wilk.
As estatísticas geradas pelo processamento dos estimadores da amostragem casual simples (Tabelas 6 e 7) indicaram que, em todas as quatro intensidades amostrais, o erro de amostragem se manteve abaixo de $10 \%$. O menor valor de erro de amostragem foi apresentado pela intensidade $1: 5,3,06 \%$, e o maior valor pela intensidade $1: 20,9,50 \%$. Pode-se considerar que a intensidade de 1:20 proporcionou uma suficiência amostral, para um erro admissível de $10 \%$, que normalmente é o máximo aceitável pelas empresas de base florestal e para os órgãos públicos florestais. No entanto, isso gerou uma considerável perda na precisão, visto que quanto maior o erro de amostragem, maior a amplitude do intervalo de confiança. A amplitude do intervalo de confiança com o processamento dos dados com intensidade 1:5 foi de $19,45 \mathrm{~m}^{3} \cdot \mathrm{ha}^{-1}$, enquanto para a intensidade de $1: 20$, este valor aumentou para $57 \mathrm{~m}^{3} \cdot \mathrm{ha}^{-1}$. 
Dantas, D. et al.

Tabela 6 - Estatísticas do processamento pelos estimadores da Amostragem Casual Simples para cada uma das quatro intensidades amostrais.

\begin{tabular}{ccccc}
\hline \multirow{2}{*}{ Resultados da ACS } & \multicolumn{4}{c}{ Intensidades } \\
\cline { 2 - 5 } & $\mathbf{1 : 5}$ & $\mathbf{1 : 1 0}$ & $\mathbf{1 : 1 5}$ & $\mathbf{1 : 2 0}$ \\
\hline Média $\left(\mathrm{m}^{3}\right)$ & 14,50 & 14,47 & 15,37 & 13,67 \\
Desvio Padrão $\left(\mathrm{m}^{3}\right)$ & 3,58 & 3,89 & 3,61 & 3,96 \\
Coeficiente de variação (\%) & 24,69 & 26,87 & 23,46 & 28,97 \\
Número de parcelas (n) & 180 & 90 & 45 & 23 \\
Erro Padrão da Média (m $\left.{ }^{3}\right)$ & 0,27 & 0,41 & 0,54 & 0,76 \\
Erro de amostragem (m $\left.{ }^{3}\right)$ & 0,44 & 0,68 & 0,90 & 1,30 \\
Erro de amostragem (\%) & $\mathbf{3 , 0 6}$ & $\mathbf{4 , 7 0}$ & $\mathbf{5 , 8 7}$ & $\mathbf{9 , 5 0}$ \\
\hline
\end{tabular}

Tabela 7 - Intervalos de confiança gerados pelos estimadores da Amostragem Casual Simples para cada uma das quatro intensidades amostrais.

\begin{tabular}{cccccc}
\hline & & \multicolumn{4}{c}{ Intervalos de confiança } \\
\cline { 3 - 5 } & & $\mathbf{1 : 5}$ & $\mathbf{1 : 1 0}$ & $\mathbf{1 : 1 5}$ & $\mathbf{1 : 2 0}$ \\
\hline \multirow{2}{*}{ Por parcela $\left(\mathbf{m}^{3}\right)$} & Volume médio & 14,50 & 14,47 & 15,37 & 13,67 \\
& Limite superior & 14,94 & 15,15 & 16,27 & 14,97 \\
& Limite inferior & 14,06 & 13,79 & 14,46 & 12,37 \\
\hline \multirow{3}{*}{ Por hectare $\left(\mathbf{m}^{3} \cdot \mathbf{h a}^{-1}\right)$} & Volume médio & 317,95 & 317,43 & 336,98 & 299,87 \\
& Limite superior & 327,68 & 332,34 & 356,76 & 328,37 \\
& Limite inferior & 308,23 & 302,51 & 317,19 & 271,37 \\
\hline \multirow{2}{*}{ Para população $\left(\mathbf{m}^{3}\right)$} & Média & 346.569 & 345.994 & 367.303 & 326.858 \\
& Limite superior & 357.166 & 362.251 & 388.867 & 357.919 \\
& Limite inferior & 335.973 & 329.738 & 345.740 & 295.797 \\
\hline
\end{tabular}

Considerando os resultados obtidos pelo processamento dos estimadores da ACS, seria plausível considerar que as intensidades 1:5 e 1:10 apresentaram desempenhos satisfatórios, por apresentar intervalos de confiança próximos entre si. Em contrapartida, as intensidades de 1:15 e 1:20 proporcionaram perdas expressivas na precisão das estimativas. No entanto, vale ressaltar que os estimadores da ACS não consideram a autocorrelação entre as parcelas e que a utilização de um interpolador geoestatístico pode aumentar a eficiência da estimativa, mesmo com uma redução na intensidade amostral (Mello et al., 2006).

As análises variográficas indicaram que os dados apresentam estrutura de continuidade espacial da variável volume de madeira por hectare, com semivariância de comportamento modelável, para todas as intensidades amostrais. Isso está de acordo, em partes, com os resultados observados por Mello et al. (2009), que detectaram continuidade espacial do volume de madeira por hectare em povoamentos de Eucalyptus spp., para as intensidades amostrais de 1:4 e 1:7. Os autores, no entanto, não observaram estrutura espacial para a intensidade amostral de uma parcela a cada 10 ha e recomendaram tratar as informações de forma independente, com a aplicação da estatística clássica.

Na Figura 2 são apresentados os semivariogramas gerados para as diferentes intensidades amostrais. Verifica-se que as linhas dos modelos esférico (azul), exponencial (preta) e gaussiano (vermelha) praticamente se confundem após a estabilização da curva. Esta diferenciação é mais perceptível nas estimativas dos efeitos pepita. 
Predição volumétrica por meio da krigagem pontual reduz o esforço de amostragem em inventários florestais pré-corte

Figura 2 - Modelos esférico (linha azul), exponencial (linha preta) e gaussiano (linha vermelha) ajustados aos semivariogramas para as intensidades amostrais 1:5, 1:10, 1:15 e 1:20.

$1: 5$

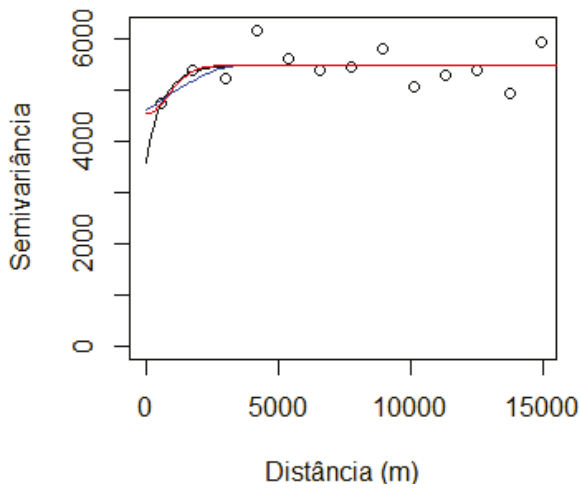

$1: 15$

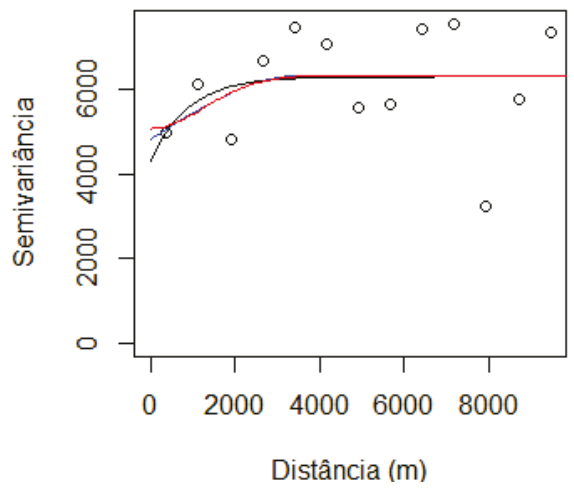

A seleção de um modelo com um bom desempenho, que represente de forma adequada as semivariâncias, especialmente nas pequenas distâncias, é importante para um melhor resultado no processo de krigagem (Nielsen e Wendroth, 2003). Por isso, além de uma análise visual do ajuste dos modelos aos diferentes semivariogramas, baseou-se também no Critério de informação de Akaike,
$1: 10$

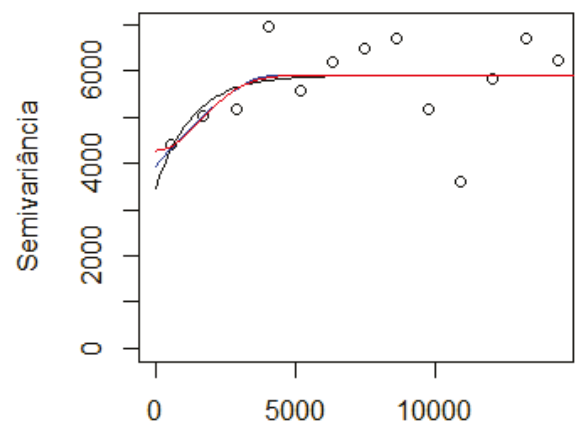

Distância (m)

$1: 20$

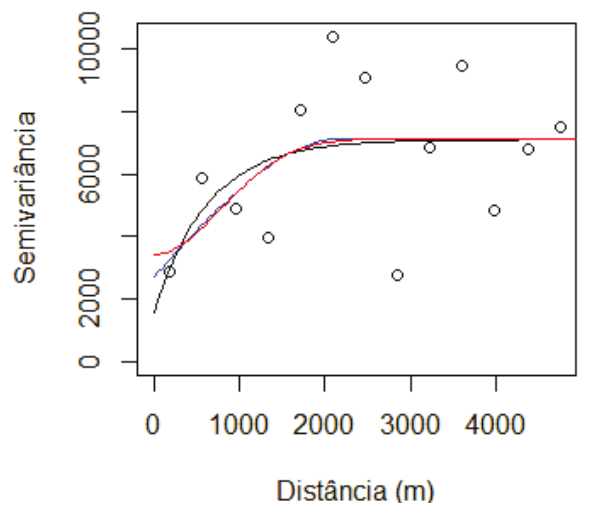

o qual indicou que, para todas as intensidades amostrais avaliadas, o modelo esférico foi o que apresentou o melhor ajuste às semivariâncias, com menores valores do AIC. Os parâmetros obtidos com o ajuste dos modelos esférico, exponencial e gaussiano, para cada intensidade amostral, estão apresentados na Tabela 8.

Tabela 8 - Estimativa dos parâmetros efeito pepita $(\tau 2)$, patamar $(\sigma 2)$ e alcance $(\theta)$ dos modelos esférico, exponencial e gaussiano ajustados aos semivariogramas da variável volume de madeira por hectare, para cada intensidade amostral.

\begin{tabular}{|c|c|c|c|c|c|}
\hline Intensidade & Modelo & $\tau^{2}$ & $\sigma^{2}$ & $\theta$ & AIC \\
\hline \multirow{3}{*}{$1: 5$} & esférico & 4606,01 & 5496,43 & 3793,31 & $-1026,3$ \\
\hline & exponencial & 3595,66 & 5483,43 & 638,42 & $-993,96$ \\
\hline & gaussiano & 4540,53 & 5481,11 & 1212,21 & -1015 \\
\hline \multirow{3}{*}{$1: 10$} & esférico & 3947,7 & 5907,81 & 4403,46 & $-993,09$ \\
\hline & exponencial & 3442,29 & 5901,98 & 1305,57 & $-960,75$ \\
\hline & gaussiano & 4269,43 & 5911 & 2230,2 & $-981,75$ \\
\hline \multirow{3}{*}{$1: 15$} & esférico & 4813,07 & 6326,62 & 3568,14 & $-927,09$ \\
\hline & exponencial & 4283,33 & 6297,88 & 883,16 & $-896,75$ \\
\hline & gaussiano & 5071,04 & 6323,79 & 1784,12 & $-918,75$ \\
\hline
\end{tabular}


Dantas, D. et al.

\begin{tabular}{cccccc}
\hline Intensidade & Modelo & $\tau^{2}$ & $\sigma^{2}$ & $\theta$ & AIC \\
\hline \multirow{3}{*}{$1: 20$} & esférico & 2702,74 & 7142,48 & 2216,61 & $-977,19$ \\
& exponencial & 1552,08 & 7095,56 & 630,17 & $-904,85$ \\
& gaussiano & 3430,01 & 7138,42 & 111,02 & $-965,85$ \\
\hline
\end{tabular}

Com o intuito de avaliar o desempenho dos modelos de semivariogramas, ajustados com diferentes intensidades amostrais, para estimar o volume por hectare, procedeu-se a uma validação preditiva. Esta validação consiste em fazer a estimativa de valores já conhecidos, mas que não foram utilizados no ajuste dos modelos.
Após a estimação dos volumes por hectare para as 40 parcelas destinadas à validação, por meio da krigagem pontual, foram calculados os erros relativos e seus desvios padrão, que são apresentados na Tabela 9.

Tabela 9 - Erros relativos da validação preditiva, gerados pelos modelos de semivariogramas ajustados para cada intensidade amostral.

\begin{tabular}{ccccc}
\hline \multirow{2}{*}{ Intensidade } & \multicolumn{4}{c}{ Erro Relativo (\%) } \\
\cline { 2 - 5 } & Mínimo & Médio & Máximo & Desvio padrão \\
\hline $1: 5$ & 0,12 & 7,33 & 18,69 & 5,83 \\
$1: 10$ & 0,07 & 7,97 & 24,57 & 6,38 \\
$1: 15$ & 0,16 & 7,71 & 21,30 & 5,44 \\
$1: 20$ & 0,83 & 8,90 & 25,34 & 6,46 \\
\hline
\end{tabular}

Verifica-se que os modelos esféricos ajustados para as quatro diferentes intensidades amostrais apresentaram desempenhos satisfatórios e próximos entre si, com erros relativos médios inferiores a $10 \%$. O menor valor foi obtido na intensidade amostral de $1: 5(7,33 \%)$, e o maior valor na intensidade de 1:20 (8,90\%). Uma diferença de erro relativo médio de $1,57 \%$. Essa diferença pode ser considerada irrisória, ao se comparar a diferença no esforço amostral em se alocar uma parcela a cada 5 ha e uma parcela a cada 20 ha, onde houve uma redução de $75 \%$ da intensidade amostral.

A Figura 3, por meio de gráficos de dispersão dos resíduos e de volumes de madeira estimados versus observados, reforça os resultados observados na Tabela 6 , indicando uma similaridade nos desempenhos dos modelos de semivariogramas com a utilização de diferentes intensidades amostrais. Os resíduos estão distribuídos em torno de zero, com uma baixa dispersão relativamente pequena.

O estimador geoestatístico, ao considerar a correlação entre as unidades amostrais, proporcionou estimativas precisas da variável volume de madeira por hectare. O desempenho dos modelos foi semelhante nas quatro intensidades amostrais avaliadas, não havendo diferença expressiva na qualidade das estimativas com a diminuição da intensidade amostral, diferentemente do que foi verificado com a utilização do estimador clássico da amostragem casual simples. Estes resultados estão de acordo com Mello et al. (2006), que modelaram a estru- tura de correlação espacial existente entre as observações da variável volume de madeira por talhão, em plantios de Eucalyptus spp. no estado de São Paulo, e observaram que o erro no método geoestatístico variou de 8 a $12 \%$ enquanto, no estimador clássico de amostragem, variou de 9 a $32 \%$.

Conforme Cochran (1977) e Thompson (1992), desconsiderando a componente espacial, ocorre superestimação da precisão, acarretando em erros de planejamento, com possíveis aumentos nos custos do inventário florestal. Assim, usando os benefícios da correlação espacial entre as parcelas, pode-se aumentar a precisão das estimativas dos inventários florestais e a intensidade de uma parcela a cada 20 ha pode atender às necessidades do inventário pré-corte e, possivelmente, também do contínuo. Com essa intensidade amostral seria possível satisfazer duas demandas importantes do planejamento florestal da empresa, que são a precisão das informações com relação ao crescimento da floresta (monitoramento) e o fornecimento de informações detalhadas essenciais para a colheita.

A diminuição da intensidade amostral reduz o custo associado ao inventário florestal, com menos gastos na alocação de parcelas, medições e processamento dos dados (Guedes et al., 2012). No entanto, os bons resultados gerados no manejo florestal não estão apenas associados aos processos de amostragem dos plantios florestais, mas também aos métodos de modelagem. 
Predição volumétrica por meio da krigagem pontual reduz o esforço de amostragem em inventários florestais pré-corte

Figura 3 - Gráficos de dispersão de resíduos e de volumes de madeira por hectare estimados versus observados, para as diferentes intensidades amostrais.

$1: 5$
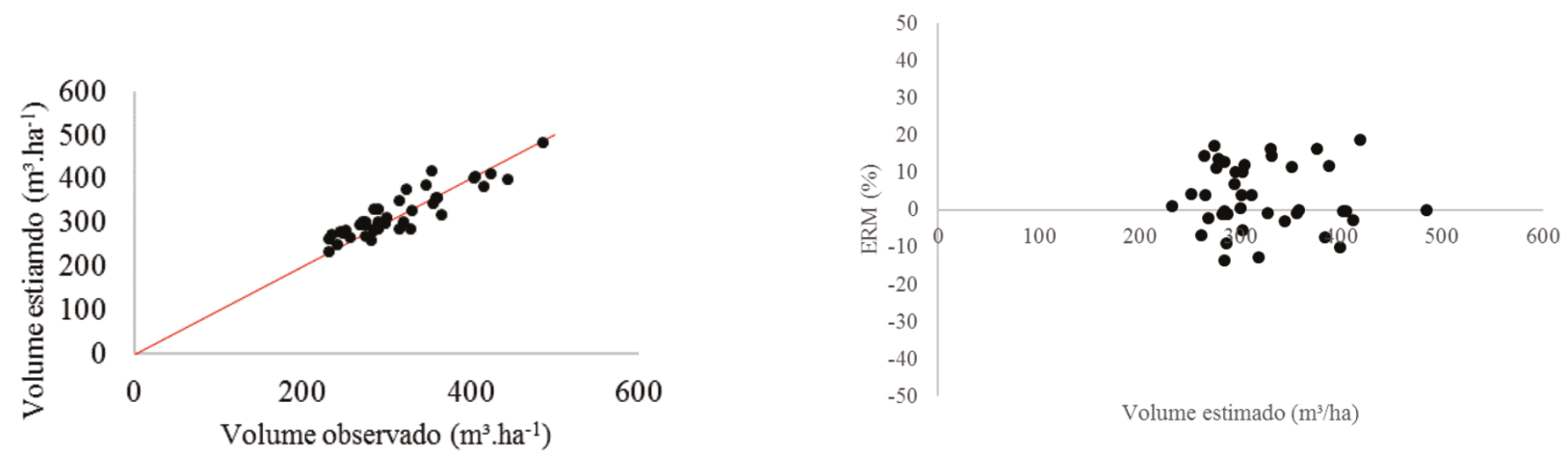

\section{$1: 10$}
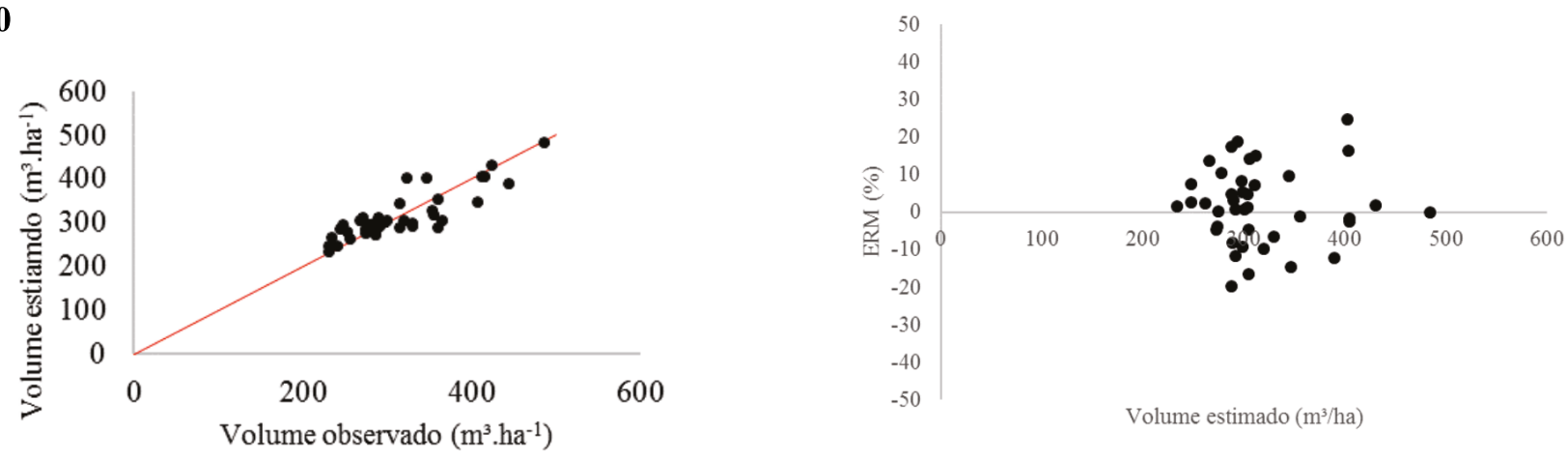

\section{$1: 15$}
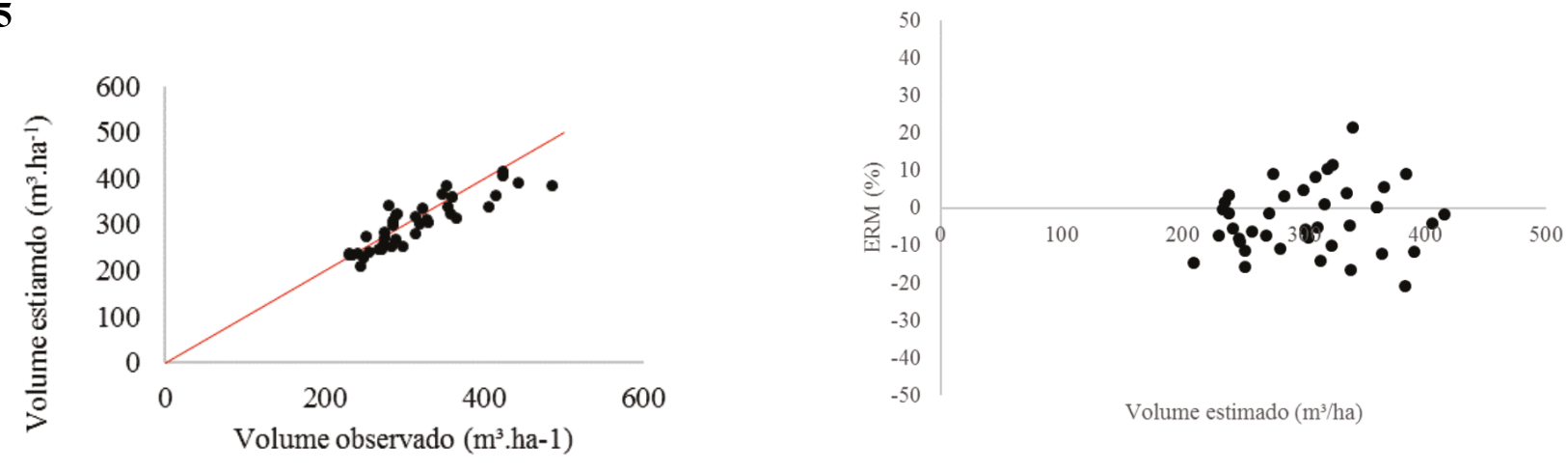

$1: 20$

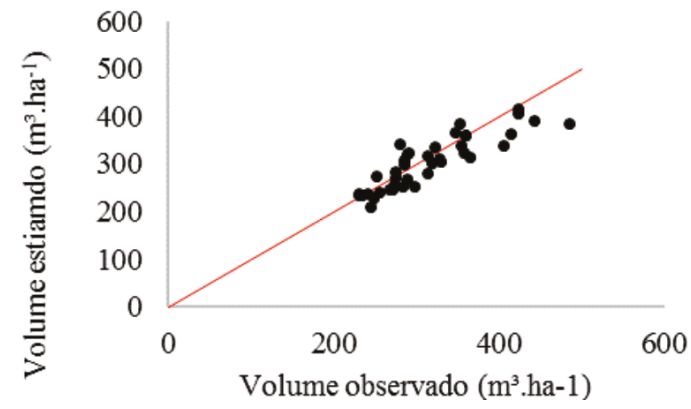

\section{Conclusão}

A geoestatística é uma técnica eficiente para estimativa da volumetria em plantios clonais de Eucalyptus spp. sob diferentes intensidades amostrais, pois permitiu uma expressiva redução da intensidade amostral em inventário pré-corte para um povoamento de Eucalyptus spp., sem perda de precisão nas predições geradas por

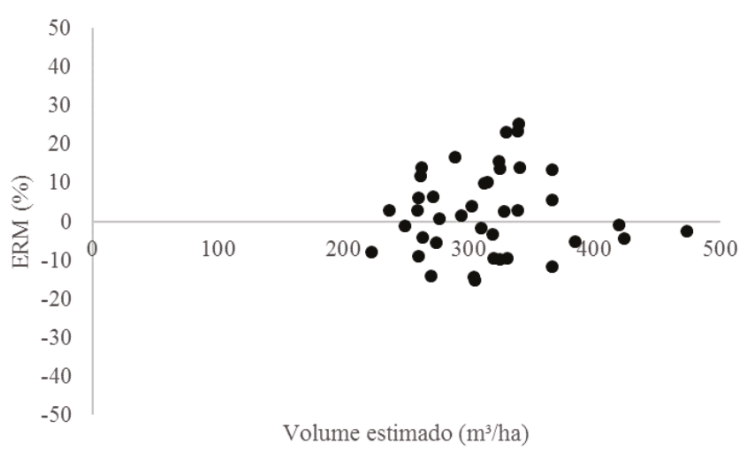

meio da krigagem pontual. Sendo o modelo esférico, que melhor se ajustou ao semivariograma, o modelo utilizado no processo de predição do volume em plantios de Eucalyptus spp. aos 8 anos de idade com erros médios inferiores a $10 \%$. 


\section{Agradecimento}

Os autores agradecem ao Programa de Pós-Graduação em Engenharia Florestal da Universidade Federal de Lavras (PPGEF-UFLA), à Coordenação de Aperfeiçoamento de Pessoal de Nível Superior (CAPES) e ao Conselho Nacional de Desenvolvimento Científico e Tecnológico pelo apoio financeiro na realização deste estudo.

\section{Referências}

Alvares, C. A.; Stape, J. L; Sentelhas, P. C.; Gonçalves, J. L. de M.; Sparovek, G. 2013. Köppen's climate classification map for Brazil. Meteorologische Zeitschrift, 22: 711-728. doi: https://dx.doi. org/10.1127/0941-2948/2013/0507.

Biase, A. G.; Santana, T. V. F. de. 2012. Geoestatística análise de dados em outro software Gamma Desing GS+. Piracicaba: Escola superior de Agricultura Luiz de Queiroz, $120 f$.

Burnham, K. P.; Anderson, D. R. 2002. Model selection and multimodel inference. 2. ed. Springer. New York.

Cochran, W. G. 1977. Sampling Techniques. 3 ed. New York.

Couto, E. G.; Stein, A.; Klamt, E. 1997. Large area spatial variability of soil chemical properties in central Brazil. Agriculture Ecosystems and Environment, 66: 139- 152. doi: https://doi.org/10.1016/S01678809(97)00076-5.

Cressie, N. 1993. Statistics for spatial data. New York: Wiley.

Dantas, D. 2019. Estimador geoestatístico e modelos de efeito misto para modelagem hipsométrica e volumétrica de povoamentos florestais. Lavras: Universidade Federal de Lavras, 120f. Dissertação Mestrado. Disponível em: http://repositorio.ufla.br/jspui/handle/1/33647.

Dantas, D.; Calegario, N.; Acerbi Júnior, F. W.; Carvalho, S. P. C.; Isaac Júnior, M. A.; Melo, E. A. 2020. Multilevel nonlinear mixed-effects model and machine learning for predicting the volume of Eucalyptus spp. trees. Cerne, 26(1): 1-10. doi: https://doi.org/10.1590/010477 60202026012668 .

Diggle, P. J.; Ribeiro Júnior, P. J. 2000. Model-based geostatistics. In: Simpósio Nacional de Probabilidade e Estatística. Associação Brasileira de Estatística.

Diggle, P. J.; Ribeiro Júnior, P. J. 2007. Model-based geostatistics. Springer. Londres.

ESRI. ArcGIS Desktop: Release 10. 2011. Redlands, CA: Environmental Systems Research Institute.

Guedes, I. C. de L.; Mello, J. M. de; Mello, C. R. de; Oliveira, A. D. de; Silva, S. T. da; Scolforo, J. R. S. 2012. Técnicas geoestatísticas e interpoladores espaciais na estratificação de povoamentos de Eucalyptus sp.. Ciência Florestal, 22: 541-550. doi: https://dx.doi. org/10.5902/198050986621.

Hernández, J.; Emery X. 2009. A geostatistical approach to optimize sampling designs for local forest inventories. Canadian Journal of Forest Research, 39: 1465-1474. doi: https://doi.org/10.1139/X09-048.

Isaaks, E. H.; Srivastava, R. M. 1989. Anintroduction to applied geostatistics. Oxford University Press. New York, NY, USA.

Journel, A. G.; Huijbregts, C. J. 1978. Mininggeostatistics. Academic Press. London, UK.

Journel, A. G.; Huijbregts, C. J. 2003. Mining geoestatistics. Academic. London, UK.
Lima, E. S.; Montanari, R.; Lovera, L. H.; Teixeira Filho, M. C. M.; González, A. P. 2017. Variabilidade espacial das propriedades dendrométricas do eucalipto e atributos químicos de um Neossolo Quartzarênico. Journal of Neotropical Agriculture, 4(1): 1-11.

Machado, S. A; Conceição, M. B.; Figueiredo, D. J. 2002. Modelagem do volume individual para diferentes idades e regimes de desbaste em plantações de Pinus oocarpa. Revista Ciências Exatas e Naturais, 4: 185-197.

Mc Bratney, A.G.; Webster, A.G. 1986. Choosing functions for semivariograms and fitting them to sampling estimates. Journal of Soil Science, 37: 617-639. doi: https://doi.org/10.1111/j.1365-2389.1986. tb00392.x.

Mello, J. M. de.; Oliveira, M. S. de; Batista, J. L. F.; Justiniano Júnior, P. R.; Kanegae Júnior, H. 2006. Uso do estimador geoestatístico para predição volumétrica por talhão. Floresta, 36: 251-260. doi: http:// dx.doi.org/10.5380/rf.v36i2.6454.

Mello, J. M.; Diniz, F. S.; Oliveira, A. D.; Scolforo, J. R. S.; Acerbi junior, F. W.; Thiersh, C. R. 2009. Métodos de amostragem geoestatística para estimativa do número de fustes e volume em plantios de Eucalyptus grandis. Floresta, 39: 157-166. doi: http://dx.doi.org/10.5380/ rf.v39i1.13735.

Mello, J. M. de. 2004. Geoestatística aplicada ao inventário florestal. Piracicaba: Universidade de São Paulo, 122f. Tese Doutorado.

Nielsen, D. R.; Wendroth, O. 2003. Spatial and temporal statistics: sampling field soils and their vegetation. Reiskirchen: Catena Verlag.

Nogueira, C. H. 2013. Análise de variância com dependência espacial sob uma abordagem geoestatística. Lavras: Universidade Federal de Lavras, 124f. Dissertação Mestrado. Disponível em: http://repositorio. ufla.br/jspui/handle/1/700.

Palmer, D. J.; Hock, B. K.; Kimberley, M. O.; Watt, M. S.; Lowe, D. J.; Payn, T. W. 2009. Comparison of spatial prediction techniques for developing Pinus radiata productivity surfaces across New Zealand. Forest Ecololy and Management, 258: 2046-2055. doi: https://doi. org/10.1016/j.foreco.2009.07.057.

R Core Team. R. 2019. A language and environment for statistical computing. Vienna: R Foundation for Statistical Computing.

Raimundo, M. R.; Scolforo, H. F.; Mello, J. M.; Scolforo, J. R. S.; McTague, J. P; Reis, A. A. R. 2017. Geoestatística Aplicada às Estimativas de Crescimento em Inventários Florestais Contínuos, Forest Science, 63(1): 29-38. Disponível em: https://doi.org/10.5849/FS-2016-056.

Ribeiro Júnior, P. J. 1995. Métodos geoestatísticos no estudo da variabilidade espacial de parâmetros do solo. Piracicaba: Escola Superior de Agricultura "Luiz de Queiroz", 99f. Dissertação Mestrado. Disponível em: https://doi.org/10.11606/D.11.2019.tde-20191108-110517.

Ribeiro Júnior, P. J.; Diggle, P. J. 2001. geoR: Apackage for geostatistical analysis. R-News, 1: 15-18.

Sakamoto, Y.; Ishiguro, M.; Kitagawa, G. 1986. Akaike information criterion statistics. Dordrecht, The Netherlands: D. Reidel. 
Predição volumétrica por meio da krigagem pontual reduz o esforço de amostragem em inventários florestais pré-corte

Santos, M. C. dos; Roveda, M.; Zanon, M. L. B.; Figueiredo Filho, A.; Roik, M.; Pacheco, J. M.; Scavinski, V. 2017. Inventário Florestal utilizando técnicas de silvicultura de precisão em povoamentos de Eucalyptus grandis Hill ex Maiden. Floresta e Ambiente, 24. doi: https://dx.doi. org/10.1590/2179-8087.082714.

Schumacher, F. X.; Hall, F. S. 1933. Logarithmic expression of timber-tree volume. Journal of Agricultural Research, 47: 719 - 734.

Sena, K. K. K.; da Silva Campos, P. S.; Rodrigues, H. C. T.; Chase, O. A.; Marques, G. T.; dos Reis Moraes, R. I.; Santos Galate, R. 2019. Análise geoestatística em povoamento de floresta nativa no município de Paragominas - Pará/Geostatistical analysis in native forest stand in the municipality of Paragominas-Pará. Brazilian Journal of Development, 5(10): 18695-18713.

Shapiro, S. S.; Wilk, M. B. 1965. An analysis of variance test for normality (complete samples). Biometrika, 52: 591-611. doi: https://dx.doi. org/10.2307/2333709.
Singh, T. P.; Das S. 2014. Predictive analysis for vegetation biomass assessment in Western Ghat region (WG) using geospatial techniques. Journal of the Indian Society of Remote Sensing, 42: 549-557. doi: https://doi.org/10.1007/s12524-013-0335-7.

Thompson, S. K. 1992. Sampling. New York: Wiley.

Viana, H.; Aranha, J.; Lopes, D.; Cohen, W. B. 2012. Estimation of crown biomass of Pinus pinaster stands and shrubland above-ground biomass using forest inventory data, remotely sensed imagery and spatial prediction models. Ecology Modeling, 226: 22-35. doi: https:// doi.org/10.1016/j.ecolmodel.2011.11.027.

Vieira, S. R. 2000. Geoestatística em estudos de variabilidade espacial do solo. In: Tópicos em ciências do solo. Viçosa, MG, Sociedade Brasileira de Ciência do Solo.

Wonnacott, T. H; Wonnacott, R. J. 1972. Introductory Statistics. 2. ed. John Wiley, New York, NY, USA. 